\title{
Relationship between salivary and serum testosterone levels in response to different exercise intensities
}

\author{
Amy R. Lane, ${ }^{1}$ Anthony C. Hackney ${ }^{1,2}$ \\ ${ }^{1}$ Endocrine Section - Applied Physiology Laboratory, Department of Exercise \& Sport Science, ${ }^{2}$ Department of \\ Nutrition, Gillings School of Global Public Health, University of North Carolina, USA
}

\begin{abstract}
The anabolic hormone testosterone ( $T$ ) continues to be researched extensively in exercise studies, with blood serum or plasma samples collected routinely. This method is considered both valid and reliable; however, the collection process can be invasive and difficult during exercise. For this reason, non-invasive sampling, such as saliva collections, is increasing in popularity. PURPOSE: To investigate the association between salivary and serum $T$ before and after various intensities of exercise to assess the validity of saliva collection for $\mathbf{T}$ measurement. METHODS: 12 endurance-trained males (Mean $\pm S D$; $\mathrm{VO}_{2 \max } 58.2 \pm 6.4 \mathrm{ml} / \mathrm{kg} / \mathrm{min}$, $22 \pm 4.6$ years, BMI 21.6 \pm 1.9 ) completed three 30-minute exercise (cycling) sessions at $40 \%$ (Low), $60 \%$ (Moderate), and $80 \%$ (High) of $\mathrm{VO}_{2 \max }$ as well as a 30-minute resting Control session on four separate days. Salivary $(\mathrm{s})$ and serum $(\mathrm{S})$ samples were assessed for $\mathrm{T}$ prior to (Pre), immediately post (Post), and 30 minutes post (30 Min Post) each session. RESULTS: Low exercise caused a significant $(p<0.05)$ increase in ST, but not $s T$, while Moderate and High exercise caused an increase $(p<0.01)$ in both ST and sT. A strong positive correlation (Spearman $\left.\left[r_{s}\right]\right)$ existed between $\mathbf{s T}$ and $\mathrm{ST}$ in the Moderate $\left(r_{s}=0.912[\mathbf{p}<0.001 ; \mathbf{n}=36 ; 12\right.$ subjects x 3 measurement times-samples] $)$, and High $\left(r_{s}=0.898[p<0.001]\right)$ sessions, but not as strong for the Low $\left(r_{s}=0.517[p=0.001]\right)$ session. CONCLUSIONS: These results suggest that sT and ST levels are strongly associated to exercise. However, the exercise-related sT and ST responses-changes were more closely aligned at Moderate and High intensities, suggesting a greater validity between the saliva and serum responses to higher intensity exercise.
\end{abstract}

Key words: Endocrine, Hormones, Men, Sport, Training

Address for correspondence:

Amy R. Lane, Human Movement Science Curriculum,

CB \# 8700 - Fetzer Hall, University of North Carolina,

Chapel Hill, North Carolina, 27599, USA,

Tel.: 919-962-5755, Fax: 919-962-0489,

E-mail: lanea@email.unc.edu

Received 17-09-2014, Accepted 30-09-2014

\section{INTRODUCTION}

Testosterone can have major impacts on multiple physiological functions as it is a key anabolic hormone. This hormone is especially important with regard to exercise and exercise training due to its role in the growth, adaptation, and maintenance of skeletal 
muscle, bone, and red blood cells. ${ }^{1}$ It is known that testosterone increases linearly with a given intensity of exercise, typically reaching a maximal level at the conclusion of the exercise. ${ }^{2}$ In research studies, it is important to measure testosterone at rest as well as during and after exercise to assess the magnitude of the actual response. Typically, total testosterone is measured in exercise studies due to the difficulty and cost of assessing the more biologically active (free) form (NB.: an alternative approach is to calculate free testosterone from total testosterone and sex hormone binding-globulin [SHBG] measurements; however, this procedure requires an additional biochemical analysis [i.e., SHBG]. ${ }^{10}$

The process of collecting blood from subjects can be problematic. A blood draw is an invasive procedure and requires an individual proficient in phlebotomy, while it may be difficult to collect samples at the desired time points (e.g., while running). Saliva measurements of hormones can provide a cost-effective collection process that introduces minimal burden to the subject. This procedure is simple and can be collected multiple times in as well as outside of a laboratory setting. Moreover, with respect to testosterone, the salivary measure captures the biologically more active free component of the hormone..$^{10}$ Thus, many researchers have concluded that the ability to utilize salivary measures can be a tremendous advantage and allow for a broader scope of research possibilities involving testosterone and exercise.

However, much of the research that has been conducted with salivary testosterone has been in resistance modalities or across a competitive training season..$^{3-6}$ Studies involving endurance or aerobic-based training are fewer in number and have typically studied hormonal responses to only low intensity exercise or a single bout of exercise, with mixed results. ${ }^{7,8,18}$ If salivary hormonal assessments are to be valid and used more frequently in exercise research, it is important to establish whether such measures are reliable and comparable to blood (serum) responses across multiple intensities of exercise in a variety of activities. Therefore, the purpose of this study was to investigate the association between serum and salivary testosterone before and after various intensities of aerobic exercise to assess the validity of saliva collection for testosterone measurement.

\section{METHODS}

\section{Subjects}

Twelve males (18-30 y) were recruited for this study. They had been exercise training a minimum of three days per week for at least 60 minutes per session for the six months leading up to the study. The exclusion criteria included: a basal diet with less than $50 \%$ of the daily caloric intake from carbohydrates (CHO), hormonal disorder history, mental illness, smoking, anabolic steroid use, and chronic use of non-steroidal anti-inflammatory drugs. Each subject was presented with and signed an informed consent as approved by the University of North Carolina Institutional Review Board.

\section{Protocol}

Each subject came to the Applied Physiology Laboratory (APL) for a total of five sessions and was instructed how to control and maintain his diet throughout the study. Prior to inclusion, a 3-day dietary record was analyzed (United States Department of Agriculture nutrition database website ${ }^{13}$ ). If the 50\% minimum $\mathrm{CHO}$ consumption was not met, further dietary instruction was given and a later day was rescheduled to verify diet prior to enrollment. Subjects reported to the APL four hours post-prandium and having consumed no caffeine or alcohol within the previous eight hours for each visit.

The initial laboratory session included all screenings and a maximal cardiopulmonary incremental exercise test $\left(\mathrm{VO}_{2 \max }\right)$ on a cycle ergometer. The remaining sessions (2-5) were for three exercise sessions: 30 minutes of cycling at a different intensity each visit (Low, Moderate, and High) and a Control resting session. The time of day for each session commencement varied between subjects, but each individual subject reported to the APL at the same time of day for their respective sessions $( \pm £ 30 \mathrm{~min}$; sessions were completed in the morning or in the afternoon although each individual completed his sessions at the same time of day. The order of the sessions was randomly assigned and was completed with at least 72 hours of rest between sessions.

\section{Screening Session}

The initial session began with the subject undergoing medical screening. Once medically approved, 
anthropometrics were collected and included: age, height, body mass, body fat $\%$ (via skinfolds). Next, the $\mathrm{VO}_{2 \max }$ protocol was initiated. A 5-minute warm-up on a stationary bike $(50 \mathrm{~W})$ followed by five minutes of stretching took place and then the subject was situated appropriately on the cycle ergometer. The 3-minute stage incremental test starting workload began at an intensity ( 50 or $100 \mathrm{~W}$ ) determined by the subject's training history and was increased by 25 or $50 \mathrm{~W}$ at each stage. This process continued until the subject reached volitional fatigue. During the $\mathrm{VO}_{2}$ test, respiratory gases were measured continuously at 15 -second intervals. Heart rate (HR) was recorded each minute and ratings of perceived exertion (RPE) were recorded at the end of each stage. A valid and reliable maximal test was determined by the subject meeting three of the following four criteria: 1) an increase in $\mathrm{VO}_{2} \leq 0.15 \mathrm{~L} / \mathrm{min}$ in response to a workload increase, 2) HR $\pm 5 \%$ of age-predicted max, 3 ) respiratory exchange ratio (RER) $\geq 1.1$, and 4) $\mathrm{RPE} \geq 18 .{ }^{14}$

\section{Exercise - Control Sessions}

Subjects came to the APL on four separate days for a minimum of 72 hours but no more than 7 days after the initial session, at the same time of day $( \pm 30$ minutes). Upon arrival, the subject completed the Rest-Q Questionnaire and, if normal scores were obtained (i.e. no undue psychological-emotional stress existed), the subject began supine rest; if scores were elevated, the session was rescheduled for a different day. Following 30 minutes rest, pre-exercise (Pre) blood and saliva samples were collected (see below). Subjects remained in a supine position during the collections. Next, a 5-minute warm-up at $15-20 \%$ of $\mathrm{VO}_{2 \max }$ was completed, followed by five minutes of stretching. The subject then mounted the bike for 30 minutes of exercise at $40 \%$ (Low), $60 \%$ (Moderate), or $80 \%$ (High) of his $\mathrm{VO}_{2 \max }$, which had been predetermined from the $\mathrm{VO}_{2 \max }$ testing results. During exercise, HR was recorded every five minutes and, to confirm appropriate intensity, respiratory gases were collected for three minutes at 7-10, 17-20, and 27-30 minute time points, and RPE was assessed at 10, 20, 30 minutes. For measures ( $\mathrm{HR}, \mathrm{VO}_{2}$, and $\left.\mathrm{RPE}\right)$ during each exercise session, an aggregate single 30-minute value was calculated for each subject. Blood and saliva samples were collected immediately upon completion of 30 minutes of exercise (Post). The subject was then allowed five minutes of active cool-down before 30 minutes of passive recovery, after which final blood and saliva samples were collected (30 Min Post). The same procedure was followed for each of the exercise sessions. The Control session was conducted in the same fashion; however, the subject completed 30 minutes of supine rest instead of exercise. However, no $\mathrm{HR}, \mathrm{VO}_{2}$, or RPE measurements were obtained during the Control session.

\section{Instrumentation}

Each subject was measured for height and mass via a stadiometer (Perspectives Enterprise, Portage, MI) and a mechanical scale (Detecto, Webb City, MO), respectively. Body fat $\%$ was determined with skinfolds in triplicate at the abdomen, chest, and thigh with the Cambridge Lange skinfold caliper (Cambridge Scientific, Cambridge, MA), and the Jackson-Pollock equation was used to predict body fat. ${ }^{12-14}$ All respiratory gases were collected using the Parvo Medics True Max 2400 Metabolic System (Parvo Medics, Salt Lake City, UT). All exercise was performed on a Lode ${ }^{\circledR}$ electronic braked cycle ergometer (Lode, Groningen, The Netherlands). Heart rates were measured using Polar heart rate monitors (Polar Model F1, Finland) and RPE was collected using Borg's scale ranging from 6-20 for perceived exertion. ${ }^{15}$

\section{Specimen Procedures}

\section{Serum}

Blood samples were collected with a 22 gauge catheter (Angiocath) from a vein at the antecubital fossa of the arm. Each sample $(3 \mathrm{~mL})$ was drawn using a $3 \mathrm{cc}$ syringe and a 25 gauge needle. Blood samples were divided and transferred immediately into EDTA (Vacutainer) and serum separator tubes (Vacutainer). Whole blood samples were immediately analyzed for hematocrit $(\mathrm{Hct})$ and hemoglobin $(\mathrm{Hb})$ in order to analyze any shift in plasma volume via the Dill and Costill method. ${ }^{16}$ Both Hct and Hb were assessed in triplicate using the microhematocrit method and the Stat-Site, WT-9 Hemoglobin Meter (Stanbio Laboratory, Boerne TX), respectively. Remaining blood samples were placed on ice until centrifuged $\left(4^{\circ} \mathrm{C}\right.$, $3000 \times \mathrm{g}$ ) and plasma and serum was transferred to cryovials and frozen at $-80^{\circ} \mathrm{C}$ for later analysis. 


\section{Saliva}

Subjects were instructed to rinse their mouths with water, spit, and allow saliva to accumulate in their mouth, and then spit directly into a propylene cup. If stimulation was necessary for secretion, subjects were asked to chew on paraffin film. The procedure was repeated until a minimum of $0.5 \mathrm{~mL}$ of saliva had been collected. Saliva was collected within five minutes, and no more, of the desired collection time. Collected samples were then stored for later analysis in cryo-freeze tubes at $-80^{\circ} \mathrm{C}$.

\section{Testosterone Analysis}

Total serum testosterone concentrations were analyzed using an enzyme-linked competitive monoclonal antibody immunoassay technique (Abnova, Taiwan; $10 \mathrm{uL}$ specimen required; sensitivity $=0.5 \mathrm{ng} / \mathrm{dL}$ ). Salivary free testosterone levels were measured using an enzyme-linked immunoassay kit (Salimetrics, State College, PA, USA; 25 uL specimen required; sensitivity $=1.0 \mathrm{pg} / \mathrm{uL}$ ). All serum and saliva subject samples were assayed in duplicates (all standards in triplicate) and all coefficients of variation within and between assays were $10 \%$ or less. $^{19}$

\section{Data Analysis}

Mean and standard deviations $( \pm \mathrm{SD})$ for all measures were calculated (STATISTICA, Statsoft, Inc., Tulsa, OK, USA). Separate, $4 \times 3$ (session $x$ time), factorial repeated measures ANOVA tests (General Linear Model) were utilized to determine if significant changes were found within serum and salivary testosterone levels. Bonferroni tests were used in the case of significant findings to identify which means were significantly different. The strength of association between serum and saliva values within each of the experiment sessions was determined using Spearman correlation $\left(r_{s}\right)$. The significance level for all analyses was set a priori at $\alpha \leq 0.05$.

\section{RESULTS}

\section{$\boldsymbol{V O}_{2 \max }$ Responses}

The physical characteristics for all subjects are displayed in Table 1. Subject responses to the $\mathrm{VO}_{2 \max }$ test were as follows: absolute and relative $\mathrm{VO}_{2 \max }=$ $3.84 \pm 0.43 \mathrm{~L} / \mathrm{min}$ and $58.2 \pm 6.4 \mathrm{~mL} / \mathrm{kg} / \mathrm{min}$, and peak
Table 1. Physical characteristics of the subjects

\begin{tabular}{lc}
\hline Characteristic & Mean \pm SD \\
\hline Age (years) & $22 \pm 5$ \\
Height $(\mathrm{cm})$ & $174.9 \pm 7.1$ \\
Body Mass $(\mathrm{kg})$ & $66.5 \pm 9.5$ \\
BMI $\left(\mathrm{kg} / \mathrm{m}^{2}\right)$ & $21.6 \pm 1.9$ \\
Body Fat $(\%)$ & $8.4 \pm 2.1$ \\
Mean CHO intake $(\%)$ & $58 \pm 4$
\end{tabular}

HR, RER, and RPE $=194 \pm 6 \mathrm{bpm}, 1.09 \pm 0.04$, and $19 \pm 1$, respectively. The duration of the tests was $15.6 \pm 2.0$ minutes.

\section{Testosterone Response}

\section{Control Session}

The hormonal responses for serum and saliva are reported in Table 2. In the Control session, the testosterone for both serum and saliva were found to not change significantly ( $\mathrm{p}>0.05)$ over the measurement times (Pre, Post, 30 Min Post).

Additionally, the respective serum and saliva testosterone values at Control Pre were compared to Pre-session values in the Low, Moderate, and High exercise sessions. These Control, Low, Moderate, and High Pre-session hormonal values for both serum and saliva were not significantly different between the sessions $(p>0.05)$. Therefore, in examining the

Table 2. Serum (ng/dL) and saliva $(\mathrm{pg} / \mathrm{mL})$ testosterone concentrations at 3 time points during 4 different sessions

\begin{tabular}{lcccc}
\hline & \multicolumn{4}{c}{ Testosterone Concentrations (Mean \pm SD) } \\
\cline { 2 - 5 } & Sample & Pre-Session & Post-Session & 30 Min Post \\
\hline \multirow{2}{*}{ Control } & Serum & $615.3 \pm 91.5$ & $601.0 \pm 97.0$ & $584.2 \pm 105.3$ \\
& Saliva & $120.2 \pm 22.8$ & $118.2 \pm 20.3$ & $119.9 \pm 24.3$ \\
Low & Serum & $632.4 \pm 83.4$ & $684.7 \pm 97.0^{*}$ & $627.9 \pm 110.6^{\dagger}$ \\
Intensity & Saliva & $117.9 \pm 24.0$ & $122.1 \pm 23.8$ & $134.4 \pm 23.7$ \\
Moderate & Serum & $599.4 \pm 118.0$ & $705.2 \pm 140.1^{*}$ & $649.4 \pm 129.0^{* \dagger}$ \\
Intensity & Saliva & $111.6 \pm 23.8$ & $148.7 \pm 30.5^{*}$ & $118.5 \pm 29.6^{\dagger}$ \\
High & Serum & $639.0 \pm 84.2$ & $789.4 \pm 111.8^{*}$ & $742.6 \pm 93.8^{* \dagger}$ \\
Intensity & Saliva & $117.2 \pm 26.8$ & $153.1 \pm 20.8^{*}$ & $150.8 \pm 24.3^{*}$ \\
\hline
\end{tabular}

*: significantly different from respective Pre-Session, $\uparrow$ : significantly different from respective Post-Session; ANOVA statistical analysis. 
exercise session responses (see below), the hormonal changes within serum and saliva within each of the exercise intensities were only compared with their own respective Pre values (and not with any Control values).

\section{Low Intensity}

Serum concentration responses indicate that the Post levels were significantly greater than both Pre $(\mathrm{p}<0.005)$ and 30 Min Post $(\mathrm{p}<0.001)$. Salivary responses revealed no significant change $(p>0.05)$ across time.

\section{Moderate Intensity}

Serum concentration at moderate intensity showed significantly increased values at Post compared to Pre $(\mathrm{p}<0.001)$ and 30 Min Post $(\mathrm{p}<0.002)$. Additionally, 30 Min Post was also significantly greater than Pre $(\mathrm{p}<0.01)$. Salivary response indicated that the Post levels were significantly greater than both the Pre $(p<0.001)$ and 30 Min Post $(p<0.001)$ measurements.

\section{High Intensity}

During High intensity exercise, Post serum testosterone concentrations were significantly greater than Pre $(\mathrm{p}<0.001)$ and 30 Min Post $(\mathrm{p}<0.02)$. Also 30 Min Post was again significantly greater than Pre $(\mathrm{p}<0.001)$ as well. Salivary concentrations were significantly increased at both Post and 30 Min Post than at Pre (both $\mathrm{p}<0.001$ ).

\section{Exercise Session Physiological Responses}

Percent changes in plasma volume $(\Delta \% \mathrm{PV})$ were calculated from Pre to both Post and 30 Min Post and are depicted in Table 3 . There was a significantly greater reduction (i.e., hemoconcentration; $\mathrm{p}<0.05$ )

Table 3. Percent changes in plasma volume $(\Delta \% \mathrm{PV})$ from Pre to both Post (immediately after exercise) and 30 Min Post (30 minutes into recovery post-exercise). *significantly greater reduction $(\mathrm{p}<0.05)$ from the Control session; ANOVA statistical analysis

\begin{tabular}{lcc}
\hline \multicolumn{3}{c}{ Percent Change Plasma Volume $(\boldsymbol{\Delta} \% \mathrm{PV})$} \\
\hline Sessions & Pre to Post & Pre to 30 Min Post \\
\hline Control & $-6.1 \pm 3.3$ & $-4.2 \pm 2.0$ \\
Low Intensity & $-11.1 \pm 4.2^{*}$ & $-5.1 \pm 4.4$ \\
Moderate Intensity & $-10.4 \pm 4.4^{*}$ & $-6.1 \pm 5.0$ \\
High Intensity & $-14.2 \pm 5.6^{*}$ & $-8.0 \pm 4.0$ \\
\hline
\end{tabular}

found in each of the exercise sessions from Pre to Post when compared to Control. No other significant changes were found.

There were significant differences $(\mathrm{p}<0.01)$ between each intensity level of exercise in $\mathrm{HR}, \mathrm{VO}_{2}$, and RPE responses (Table 4). The actual exercise intensity at each session was slightly higher than intended by $\sim 5 \%$. These intensities, however, did not overlap, as shown in the data in Table 4.

\section{Correlations}

Individual correlation coefficients were calculated for serum vs. saliva testosterone values within each of the experiment sessions. These coefficients were as follows; Control $-r_{s}=0.859(\mathrm{p}<0.001, \mathrm{n}=36[12$ subjects x 3 measurement times-samples]); Low Intensity $-r_{s}=0.517(\mathrm{p}=0.001)$, Moderate Intensity $-r_{s}=0.912(\mathrm{p}<0.001) ;$ High Intensity $-r_{s}=0.898$ $(\mathrm{p}<0.001)$.

\section{DISCUSSION}

The primary purpose of this study was to investigate the association between serum and salivary testosterone before and after various intensities of exercise to assess the validity of saliva collection for testosterone measurement (i.e., saliva responses being representative of serum responses). This was accomplished through collecting serum and saliva specimens simultaneously at Pre, Post, and 30 Min Post exercise sessions at intensities of $40 \%, 60 \%$, and $80 \%$ of $\mathrm{VO}_{2 \max }$ and analyzing these specimens for testosterone concentrations.

The serum testosterone levels measured across all

Table 4. Mean \pm SD results of select variable measures from each exercise session $(n=12)$. Aggregate overall response over the 30 minutes of exercise

\begin{tabular}{lccc}
\hline Measure & $\begin{array}{c}\text { Low } \\
\text { Intensity } \\
\mathbf{( 4 0 \% )}\end{array}$ & $\begin{array}{c}\text { Moderate } \\
\text { Intensity } \\
\mathbf{( 6 0 \% )}\end{array}$ & $\begin{array}{c}\text { High } \\
\text { Intensity } \\
\mathbf{( 8 0 \% )}\end{array}$ \\
\hline Workload (W) & $95.0 \pm 17.0$ & $145.4 \pm 18.8$ & $181.9 \pm 26.1$ \\
Heart Rate (bpm) & $127 \pm 14$ & $156 \pm 14$ & $180 \pm 8$ \\
$\mathrm{VO}_{2}(\mathrm{~mL} / \mathrm{kg} / \mathrm{min})$ & $26.3 \pm 3.3$ & $37.8 \pm 4.3$ & $49.4 \pm 4.6$ \\
$\% \mathrm{VO}_{2 \max }$ & $45.3 \pm 5.0$ & $65.2 \pm 7.7$ & $85.0 \pm 5.8$ \\
$\mathrm{RPE}$ & $10 \pm 2$ & $13 \pm 2$ & $16 \pm 1$ \\
\hline
\end{tabular}


sessions were in line with values reported in previous studies. ${ }^{1}$ Similarly, the salivary response was also reflective of previously reported values in conjunction with bouts of exercise. ${ }^{11}$ This agreement with previous literature supports the validity of the specimen collection and the biochemical analysis of the specimens. ${ }^{17}$ Relative to the serum testosterone responses, it is important to note that a small portion of the changes observed were due to hemoconcentration effects as plasma volume shifted from the vascular space, an expected occurrence. ${ }^{14,16,19}$

Relative to exercise, the changes and associations observed in/between serum and saliva testosterone appear more closely aligned in Moderate and High intensities of exercise. However, the finding that Low intensity exercise saliva and serum responses did not mirror one another was unexpected. This finding may be due to an ineffective stimulus of the salivary response at a Low intensity (i.e., an insufficient concentration gradient for free testosterone movement from the blood). This notion is supported by the serum data. That is, the magnitude of the blood testosterone changes with exercise were the smallest in the Low intensity exercise, and increased substantially with the greater exercise intensities, hence increasing the concentration gradient. ${ }^{19}$ Obviously this explanation raises the question as to why the Control session responses-association of saliva and serum testosterone were in such strong agreement. In this case, the subjects were in an extended period of quiet rest ( $\sim 90$ minutes) without any perturbations to change the hormone levels in the blood. This extended period may have resulted in the blood and saliva levels of testosterone being in equilibrium and highly reflective of one another.

There were several limitations and delimitations within this study that should be noted. The sample size was small $(\mathrm{n}=12)$; however, there was sufficient power $(\beta=0.80 ; \alpha=0.05)$ to detect significant differences in the respective salivary and serum testosterone responses. The physical characteristics of the subjects as young, healthy, trained males may impede the ability to generalize the findings to a broader population. Furthermore, subjects completed sessions at different times of the day; although each individual completed his sessions at the same time of day, some subjects completed them in the morning and others in the afternoon (hence the diurnal effects were consistent within each subject). In our sampling for saliva, while procedures were thoroughly explained to the subjects, mouths were not inspected prior to collection to confirm no presence of blood or other conflicting debris which may have impacted results. ${ }^{20}$ However, all saliva values were within normal limits. Finally, salivary testosterone measures the free form as opposed to the total testosterone as was measured in serum. Although most authors accept that free testosterone mirrors total testosterone, in our study this was not the case in several instances. ${ }^{21}$ On the other hand, key strengths of the study include the stringent controls in dietary consumption, training habits, external stressors, and other physical activity during the study, as well as the standardized and controlled testing protocols. All the aforementioned factors are known to significantly influence testosterone levels and measurements.

In conclusion, taken collectively, the findings from this study indicate that a strong association exists between saliva and serum testosterone responses at rest and in response to moderate and high intensity levels of exercise. This suggests that salivary measures of testosterone may be a viable alternative to blood draws in exercise-based research, provided the intensities being studied are sufficiently high and measurement situations are controlled meticulously.

\section{REFERENCES}

1. Zitzmann M, Nieschlag E, 2001 Testosterone levels in healthy men in relation to behavioral and physical characteristics: facts and constructs. Eur J Endocrinol 144: 183-197.

2. Wilkerson JE, Horvath SM, Gutin B, 1980 Plasma testosterone during treadmill exercise. J Appl Physiol Respir Environ Exerc Physiol 49: 2492-2453.

3. Cadore E, Lhullier F, Brentano M, et al, 2008 Correlations between serum and salivary hormonal concentrations in response to resistance exercise. J Sports Sci 26: 1067-1072.

4. Beaven CM, Gill ND, Cook CJ, 2008 Salivary testosterone and cortisol responses in professional rugby players after four resistance strength exercise protocols. J Strength Cond Res 22: 426-432.

5. Kraemer WJ, Loebel CC, Volek JS, et al, 2001 The effect of heavy resistance exercise on the circadian rhythm of salivary testosterone in men. Eur J Appl Physiol 84: 13-18.

6. Beaven CM, Gill ND, Ingram JR, Hopkins WG, 2011 
Acute salivary hormone responses to complex exercise bouts. J Strength Cond Res 25: 1072-1078.

7. Lac G, Berthon P, 2000 Changes in cortisol and testosterone levels and $\mathrm{T} / \mathrm{C}$ ratio during an endurance competition and recovery. J Sport Med Phys Fit 40: 139-144.

8. Tremblay MS, Copeland JL, Van Helder W, 2005 Influence of exercise duration on post-exercise steroid hormone responses in trained males. Eur J Appl Physiol 94: 505-513.

9. Crewther BT, Lowe TE, Ingram J, Weatherby RP, 2010 Validating the salivary testosterone and cortisol concentration measures in response to short, high-intensity exercise. J Sports Med Phys Fitnes 50: 85-92.

10. Vittek J, L'Hommedieu DG, Gordon GG, Rappaport SC, Southren AL, 1985 Direct radioimmunoassay (RIA) of salivary testosterone: correlation with free and total serum testosterone. Life Sci 37: 711-716.

11. U.S. Department of Agriculture, Agricultural Research Service. Nutrient Data Laboratory Home Page. http:// www.ars.usda.gov/ba/bhnrc/ndl. Accessed 28 February 2010 .

12. American College of Sports Medicine 2000 Guidelines for Exercise Testing and Prescription, $6^{\text {th }}$ edition. Baltimore, MD: Williams and Wilkins: 16-23; 43-47; 56-66.

13. Brooks GA, Fahey TD, White TP, Baldwin KM, 2000 Exercise Physiology: Human Bioenergetics and Its
Applications. Mountain View. Mayfield Publishing Company; pp,186-199.

14. Powers SK, Howley ET, 2004 Exercise Physiology: Theory and Application to Fitness and Performance. New York: McGraw-Hill; pp, 81-83.

15. Borg G, 1970 Perceived exertion as an indicator of somatic stress. Scand J Rehabil Med 2: 92-98.

16. Dill DB, Costill DL, 1974 Calculation of percentage changes in volumes of blood, plasma, and red cells in dehydration. J Appl Physiol 37: 247-248.

17. Granger DA, Shirtcliff EA, Booth A, Kivlighan KT, Schwartz EB, 2004 The "trouble" with salivary testosterone. Psychoneuroendocrinology 29: 1229-1240.

18. Duclos M, Corcuff JB, Rashedi M, Fougere V, Manier G, 1996 Does functional alteration of the gonadotropic axis occur in endurance trained athletes during and after exercise? A preliminary study. Eur J Appl Physiol 73: 427-433.

19. Hackney AC, Viru A, 2008 Research methodology: issues with endocrinological measurements in exercise science and sports medicine. J Athl Train 43: 631-639.

20. Schwartz EB, Granger DA, 2004 Transferrin enzyme immunoassay for quantitative monitoring of blood contamination in saliva. Clin Chem 50: 654-656.

21. Wood P, 2009 Salivary steroid assays - research or routine? Ann Clin Biochem 46: 183-196. 